\title{
REVIEW
}

\section{Globalization of Alzheimer's disease clinical trials}

\author{
Jeffrey Cummings ${ }^{1 *}$, Robert Reynders² and Kate Zhong'
}

\begin{abstract}
Alzheimer's disease (AD) therapies are increasingly being tested in global clinical trials. A search of ClincalTrials.gov revealed that of 269 currently active trials, $28 \%$ are currently being conducted in the United States; the majority of trials and the majority of trial sites are ex-US. The US has the largest number of trial sites of any single country; cumulatively, nearly half of all sites are outside the US. The US conducts more trials in all phases of drug development but has a greater proportion of phase 3 trials. The increasing importance of global participants in clinical trials emphasizes the importance of considering the ethnic and international factors that may influence trial outcome. The International Conference on Harmonization guidelines divide ethnic factors that may affect drug development into intrinsic and extrinsic influences. These include language, cultural factors, educational levels, the general level of health and standard of care, as well as nutrition and diet. Ethnic influences on pharmacokinetics are known for some metabolic pathways. The biology of AD may also differ among the world's populations. The frequency of the apolipoprotein e4 allele, a major risk factor for AD, differs internationally. Genetic variations might also affect inflammatory, excitotoxic, and oxidative components of AD. Diagnostic standards and experience vary from country to country. Levels of practitioner training and experience, diagnostic approaches to $A D$, and attitudes regarding aging and AD may differ. Experience and sophistication with regard to clinical trial conduct also vary within and between countries. Experience with conducting the necessary examinations, as well as the linguistic and cultural validity of instrument translations, may affect trial outcomes. Operational and regulatory aspects of clinical trials vary and provide important barriers to seamless conduct of multiregional clinical trials. Collection and testing of biological samples, continuous provision of drug substance, and protection of the integrity of supply lines may be difficult in some international circumstances. Attention to these potential influences on clinical trials will determine the success of global drug development programs and the utility of global trials for developing new AD therapeutics.
\end{abstract}

Alzheimer's disease (AD) is a growing global epidemic crisis. Currently, 35.6 million people are living with dementia worldwide; the number is expected to increase to 65.7 million in 2030 and 115.4 million in 2050 [1]. These staggering numbers require the development of meaningful interventions that will delay the onset, slow the progression or improve the symptoms of patients suffering from this progressive neurodegenerative disorder. Many promising agents show efficacy in preclinical models of AD and must be tested for efficacy in double-blind, placebo controlled clinical trials.

More AD clinical trials are conducted in the United States (US) than in any other single country, but most trials now have a significant number of international sites

*Correspondence: cumminj@ccf.org

${ }^{1}$ Cleveland Clinic Lou Ruvo Center for Brain Health, Las Vegas, Nevada. ${ }^{2}$ Tuoro

School of Osteopathic Medicine, Las Vegas, Nevada 89106, USA.

Full list of author information is available at the end of the article and there are numerous compelling reasons to consider incorporating international sites in $\mathrm{AD}$ trials. Most clinical trials fail to recruit the necessary numbers of patients within the anticipated timeframe [2]. Recruitment must extend globally in order to facilitate patient recruitment and enable trials to be completed in acceptable periods. The presence of large numbers of patients, many of whom are treatment naïve, enhances the attractiveness of international clinical trial sites. In addition, trials conducted outside of the US are typically less expensive, providing another motivation for ex-US drug development. There are an increasing number of well trained scientists internationally, and laws and regulatory provisions make using foreign sites an attractive alternative [3]. Finally, most patients with dementia live in the developing world [4] and the growing size of international pharmaceutical markets [5] attracts sponsors to conduct studies in countries where pharmaceutical sales may be pursued. With the growth of globalization of clinical trials, there are now more studies conducted collectively outside of the US than within the 
US [6]. Globalization of trials is an increasing trend and will continue into the foreseeable future [7].

There are many challenges to reliable drug development in global settings and some of the recent failures in $\mathrm{AD}$ drug development may be attributable in part to inadequate attention to these issues [8]. Here we discuss the aspects of drug development to be considered when implementing global clinical trials for $\mathrm{AD}$ therapies.

\section{Current global distribution of Alzheimer's disease trials}

A search of the ClinicalTrials.gov registry using the term 'Alzheimer's', was refined to 'trials funded by industry'. This search yielded 475 studies as of 3 March 2011. From these results, studies were included if they had a status of 'recruiting', 'active, not recruiting', or 'enrolling by invitation'; 269 studies met the inclusion criteria.

The number of locations listed for each study in each of 14 regions was analyzed. The regions were: US, Canada, South America, Mexico/Central America, Middle East, Africa, Western Europe, Eastern Europe, Russia, India/ Southeast Asia, China, Korea, Japan and Australia/New Zealand. Sites in Taiwan and Hong Kong were included with China. Western Europe was defined as the United Kingdom, Ireland, Iceland, Portugal, Spain, France, Benelux, Denmark, Norway, Sweden, Finland, Germany, Austria, Switzerland, and Italy. The group of Eastern European countries excluded Russia, which was its own region. Turkey was included with the Middle East. Most of the trials were for pharmaceutical agents; a few (11) are for evolving diagnostic devices or procedures.

Of the 269 studies meeting the search criteria, 76 (28\%) are being conducted in the US whereas a majority of trials are occurring outside the US (Table 1). Western Europe is host to 43 studies (16\%); other countries each had fewer trails but collectively accounted for a majority of trials. Forty-nine (18\% of all trials; $64 \%$ of all US trials) are being conducted only in the US; 27 (10\% of all trials; $35 \%$ of US trials) are multinational. The US has the largest number of trial sites (1,937 total for all sites in all trials; many sites participate in several trials and the total includes these sites for every trial), contributing $48 \%$ of all active trial sites for all trials. Europe had the second largest number of active trial sites $(921 ; 23 \%)$. As in the total number of trials, there were more trial sites $(52 \%)$ outside the US than within.

The global distribution of trials by phase is shown in Table 2. ClinicalTrials.gov lists 25 phase 1,49 phase 2, 30 phase 3 , and 15 phase 4 AD trials. Phase 1 trials may be under-represented in the data base since some foreign phase 1 trials are not conducted under an investigational new drug application [7]. Of the phase 1 trials listed, most are conducted in the US and Western Europe (20 of 25 are conducted in these two regions). Phase 2 and
Table 1. Current global distribution of Alzheimer's disease trials funded by industry

\begin{tabular}{lcc}
\hline Regions & $\begin{array}{c}\text { Total number of } \\
\text { locations by region }\end{array}$ & Total clinical trials \\
\hline United States & 1,937 & 76 \\
Western Europe & 921 & 43 \\
Canada & 171 & 23 \\
Eastern Europe & 238 & 22 \\
Australia/New Zealand & 113 & 20 \\
Japan & 305 & 17 \\
South America & 87 & 13 \\
Korea & 31 & 12 \\
Russia & 91 & 11 \\
Middle East & 48 & 10 \\
China & 27 & 9 \\
Africa & 33 & 6 \\
Mexico/Central America & 19 & 5 \\
India/Southeast Asia & 11 & 2 \\
\hline
\end{tabular}

Trial sites typically participate in more than one trial and may be represented several times in the 'Total number of locations by region' column.

phase 3 trials are conducted worldwide; most countries except Russia have approximately equal numbers of phase 2 and 3 trials. Russia has a predominance of phase $2 \mathrm{AD}$ trials (7 of 11 trials conducted in Russia). The US is involved in $52 \%$ of the listed phase 1 trials, $59 \%$ of phase 2 trials, and $73 \%$ of phase 3 trials.

\section{Aspects to be considered in globalized Alzheimer's disease trials}

The International Conference for Harmonization (ICH) guideline E5 [9] provides a framework for discussing ethnic influences in clinical trials relevant to globalized drug development programs. The guidelines divide ethnic influences into intrinsic and extrinsic factors. Table 3 provides a summary of major dimensions of drug development to be considered when planning global AD clinical trials.

\section{Host factors that may affect disease progression}

The Human Development Index, which consists of life expectancy, education and per capita gross national income (as an indicator of standard of living), provides insight into differences in patient conditions throughout the world. Human Development Index scores vary from 0 (very poor) to 1 (very good). North America and Australia generally have values of 0.9 and above; Russia 0.7 to 0.749 ; China 0.65 to 0.69 ; India 0.55 to 0.59 ; parts of Africa may be as low as under 0.3 [10]. Studies of specific health indices across countries also provide examples of international variability. For example, a comparison of cardiovascular health of individuals included in the US 
Table 2. Distribution of Alzheimer's disease trials by phase

\begin{tabular}{|c|c|c|c|c|}
\hline & Phase 1 & Phase 2 & Phase 3 & Phase 4 \\
\hline United States ${ }^{\mathrm{a}}$ & 13 & 29 & 22 & 7 \\
\hline Western Europe & 7 & 17 & 15 & 1 \\
\hline Canada & 0 & 10 & 11 & 1 \\
\hline Eastern Europe & 0 & 10 & 11 & 0 \\
\hline Australia/New Zealand & 2 & 6 & 10 & 1 \\
\hline Japan & 1 & 4 & 9 & 2 \\
\hline South America & 0 & 5 & 7 & 0 \\
\hline Korea & 0 & 3 & 5 & 1 \\
\hline Russia & 0 & 7 & 4 & 0 \\
\hline Middle East & 1 & 3 & 3 & 3 \\
\hline China $^{b}$ & 1 & 2 & 4 & 1 \\
\hline Africa & 0 & 1 & 4 & 0 \\
\hline Mexico/Central America & 0 & 2 & 3 & 0 \\
\hline India/Southeast Asia & 0 & 0 & 1 & 1 \\
\hline
\end{tabular}

aThe United States had one trial listed as phase I/II. ${ }^{\mathrm{b} C h i n a}$ had one trial listed as phase IIIIII.

National Health and Nutrition Examination Survey with the health measures of those included in the Nutrition and Health Survey in Taiwan revealed differences between the two ethnic groups in left ventricular hypertrophy, serum lipids, obesity and smoking status [11]. Such international differences could translate into differences in cerebrovascular co-morbidity, cognitive reserve, and adverse event vulnerability. Health status measures could be incorporated in trial analyses to address variability introduced by international health disparities.

Education levels also vary substantially from country to country. The average education level in the US is 12 years; Japan has an average education level of 9.5 years; United Kingdom 9.4; France 7.9; Italy 7.2; Thailand 6.5; China 6.4; India 5.1; and Brazil 4.9 [12]. Many countries included in global clinical trials have less than half the educational level of US trial participants. In a validity study of the Chinese version of the Alzheimer's Disease Assessment Scale - Cognitive portion (ADAS-cog), for instance, $35 \%$ of the trial subjects had five or fewer years of education [13]. This contrasts with the US, where participants have an average of 14 years of education in recent trials [14]. Individuals with lower levels of education tend to progress more slowly in the course of AD [15]. Persons with higher levels of education progress more rapidly, have higher levels of neuritic plaques at autopsy, and have more severe abnormalities on fluorodeoxyglucose positron emission tomography and amyloid imaging than those with lower levels of educational achievement [16-19]. Placebo groups would decline more slowly and larger number of individuals would be
Table 3. Factors that may effect global Alzheimer's disease clinical trials

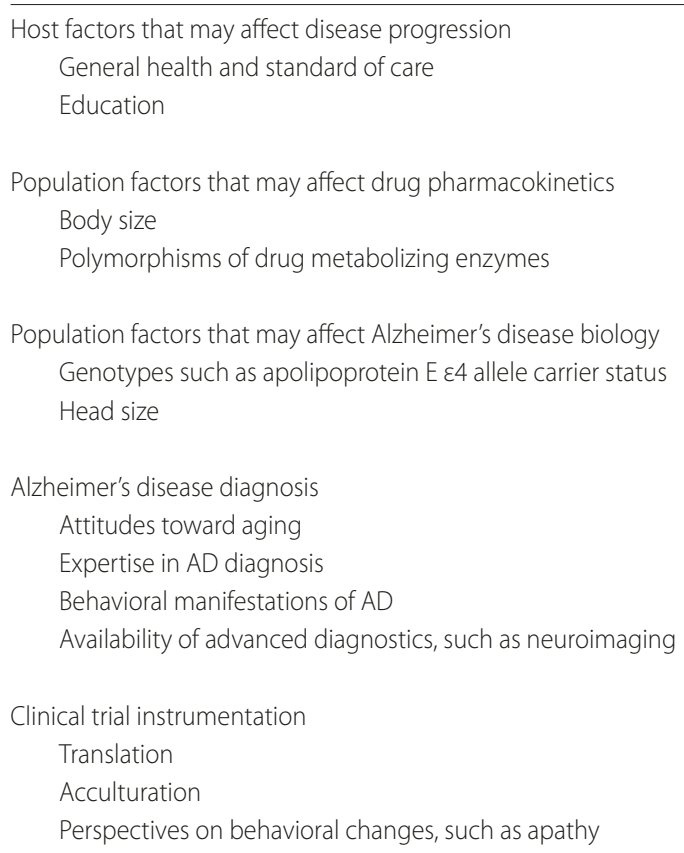

required to show a drug-placebo difference in a poorly educated population compared to a well educated population. Effects of education on disease progression in different regions have not been investigated, and educational level should be considered as a potentially 
important source of variance in disease progression in global trials. The level of education in the proposed population would need to be known to model the magnitude of this effect for specific trials.

Exercise levels may also vary among global populations and exercise has been shown to influence amyloid-beta protein abnormalities in cognitively normal older persons. Individuals with higher levels of exercise have lower levels of amyloid on amyloid imaging and higher (less abnormal) levels of amyloid levels in the cerebrospinal fluid [20]. This influence should be considered when populations with markedly different exercise levels are included in global prevention trials of agents targeting beta-amyloid mechanisms.

\section{Population factors that may influence drug pharmacokinetics}

Differences in body size would be expected to contribute to differences in brain exposure levels when patients of different sizes are given similar doses of drugs. The average height in Sweden, for example, is 1.815 meters, whereas the average height in the Philippines is 1.634 meters, a 20 centimeter difference with corresponding anticipated differences in drug exposures [21]. In Japan, where body size tends to be smaller than in Western nations, two-thirds of new molecular entities approved between 2003 and 2005 had standard doses that differed from those approved in the US and Europe [22].

Obesity likely may affect drug metabolism and distribution. Obesity is more common in Western populations. Lipophilic drugs will have disproportionately large volumes of distributions in obese and overweight individuals [23]. Such differences may affect brain exposures and both efficacy and adverse event occurrence.

Polymorphisms that may influence drug metabolism, such as those governing cytochrome P450 enzymes, may also vary substantially across ethnic groups and could contribute importantly to differences in drug metabolism, central nervous system drug exposure, and drug response $[24,25]$. There are more slow metabolizers in Asian populations, possibly leading to higher exposure levels with contrasting efficacy and side effect profiles among participants in global trials.

Concomitant approved drugs taken by patients in trials may also affect trial results through drug-drug and drugdisease interactions. Different approaches to managing medical conditions prevail globally and average doses in clinical use may vary. Japanese physicians, for example, tend to use small doses of several medications to manage medical illnesses; European doctors tend to gradually titrate single medications upward; and US physicians are more likely to press the dose upward until toxicity occurs and then titrate downward [23]. These practice patterns result in smaller doses of medications in Japan,
Table 4. Characteristics of compounds less sensitive to ethnic influences and better candidates for global trials

Linear pharmacokinetics

Wide therapeutic dose range

Minimal metabolism or not metabolized by enzymes subject to genetic influences

High bioavailability

Little inter-subject variability in bioavailability

Low protein binding

Limited potential for drug-diet interactions

Limited potential for drug-drug interactions

Limited potential for abuse or inappropriate use

Not a prodrug

Adapted from $[1,3,23]$.

intermediate doses in Europe, and higher doses in the US. This creates an opportunity for differing drug-drug interactions across regions.

Some compounds may be less sensitive to ethnicrelated influences and are better candidates for global clinical trials. Table 4 summarizes the characteristics of compounds less sensitive to ethnic influences.

Biological therapeutic agents may be subject to somewhat different global influences than small molecule drugs. Asians, for example, have higher rates of exposure to infections and a different complement of circulating antibodies; they are less likely to have been included in national immunization campaigns and therefore have more naïve immunological backgrounds from an iatrogenic perspective [23].

\section{Population factors that may affect Alzheimer's disease biology}

Genetic diversity also creates differences in the biology of $\mathrm{AD}$ relevant to clinical trials. The apolipoprotein $\mathrm{E} \varepsilon 4$ allele (APOE ع4) varies from a high frequency of 0.221 in Oceania to a low frequency of 0.068 in India [26]. APOE $\varepsilon 4$ carrier status influences the rate of progression of both cognitive and functional decline in patients with mild cognitive impairment and prodromal $\mathrm{AD}[27,28]$. Ethnic differences in receptor sensitivity and pathway modulation have been insufficiently explored but also could affect drug efficacy in diverse populations. This variability across ethnicities and regions could influence rate of disease progression and affect the sample size needed to observe a drug-placebo difference with an efficacious agent.

Head size and brain size reflect cognitive reserve and have an important interaction with disease state. Head size reflects brain size and neuronal number. Smaller head size has been associated with more rapid progression of AD [29]. Similarly, smaller head size is associated 
with greater cognitive impact of brain atrophy compared to persons with larger heads [30]. Persons with smaller body size have correspondingly smaller head sizes and patients from countries with smaller citizens might progress more rapidly in trials. More comprehensive modeling of this effect is needed and collection of empirical data is warranted.

Diet and nutrition might also contribute to differences in therapeutic response, side effect vulnerability, and disease progression. Populations with high levels of antioxidants in the diet might reduce oxidative injury, while those with high levels of salt or animal fats may be more vulnerable to hypertension, cardiovascular disease and central nervous system co-morbidity.

\section{Approaches to Alzheimer's disease diagnosis that may differ among countries}

Attitudes toward aging dictate the extent to which cognitive decline is considered abnormal and may affect rates of referral for evaluation and trial participation. Asian countries place less emphasis on the importance of memory in aging, and AD is less likely to be perceived as an abnormality [4]. In countries where aging and dementia are not distinguished, referral for cognitive assessment is typically later in the disease course when cognitive decline is more severe; consultation may be triggered by behavioral changes rather then cognitive or memory complaints [4,31]. Dementia diagnosis will be delayed in these settings and behavioral disturbances are more common in conjunction with AD diagnoses.

In some countries, the high frequency of hypertension, cardiovascular, and cerebrovascular disease will increase the proportion of patients with mixed dementia syndromes or comorbid central nervous system pathology [32].

Clinical phenomenology may be affected by international setting. For example, rates of apathy as measured by the Neuropsychiatric Inventory (NPI) are lower in Asian compared to US populations [33], likely attributable to differences in expectations of activity levels and the value placed on serenity in elderly persons.

Clinicians vary widely in the extent to which they use standardized rating scales and collect systematic information on AD in their clinical practices [34]. Differences in practice may translate into differences in understanding the variability in presentations of $\mathrm{AD}$ and their impact on diagnosis.

\section{Aspects of clinical trial instrumentation that may affect trial outcomes}

Nearly all widely used clinical trial instruments for AD were developed in North America, and they may not be well suited for use in other countries, especially those with different cultural heritages. Using the same instruments in all sites of a global clinical trial is required for data uniformity and analysis, but the quality of the data collected may be affected by differing cultural and national influences. Elderly in many developing countries have low levels of reading and health literacy, poor testtaking skills, and higher levels of test-related anxiety [4]. If skills improve and anxiety declines with multiple test sessions, both the active treatment group and the placebo group will exhibit substantial improvement, possibly obscuring a drug-placebo difference.

There have been numerous local translations of clinical trial instruments, often with little attempt at harmonization or consideration of the impact of the differences on trial outcomes. In the Hindi version of the MiniMental Status Examination, for example, 16 of the items were changed to make it culturally appropriate [4]. Even within the US, substantial administration and scoring variances have been documented for the ADAS-cog [35] and the NPI [36]. These differences are exaggerated in global trials.

Normative data for trial instruments are often lacking in cultures where clinical trials are conducted and cutoff scores for study entry as well as expected performances for healthy elderly persons may be difficult to extrapolate from Western experience. This can result in entry of noncompromised patients into prodromal $\mathrm{AD}$ and $\mathrm{AD}$ dementia trials because of culturally and educationally appropriate low scores.

Trial data for the Clinical Dementia Rating (CDR), Activities of Daily Living Scale, and the NPI are collected from caregivers. The role of the care partner may be more straightforward in Western countries than in many developing nations. Patients tend to live at home in extended families with multiple caregivers. Many families, even those of modest financial means, employ domestic maids who may speak different dialects or even different languages from the patients [4]. These individuals are responsible for the majority of the daily care of the affected person. How best to collect information under these circumstances and how best to define 'caregiver' can be challenging. Many indigenous persons have extremely simple life styles unencumbered by utilities and conveniences; the usual trial instruments will not effectively quantify functional changes in these circumstances.

Biological measures may also be differentially acceptable across international trial sites. Asian patients are reported to be more reluctant to undergo invasive procedures and less likely to participate in trials including lumbar puncture [4].

\section{Clinical trial conduct practices that may vary among countries}

Clinicians and trial sites vary widely with regard to their experience in conducting AD clinical trials. In a review of 
their proprietary database of 7,000 raters, one ratertraining company found that $57 \%$ of raters had more than 5 years' experience in administering the ADAS-cog and $27 \%$ had less than 1 year's experience [37]. Among raters in China included in a recent trial, $40 \%$ of raters had administered the ADAS-cog fewer than five times. Lack of experience can lead to greater score variability and more difficulty demonstrating a drug-placebo difference of an effective compound.

Clinical trial conduct includes training the sites on how to administer the assessment instruments and monitoring sites for appropriateness of trial data collection. Many investigator meetings focus on proper scoring of instruments based on videotaped interviews by experts. Investigators and research assistants may have limited opportunity to administer the examinations under supervised circumstances. Reliability studies of the Clinical Dementia Rating show that at least five administrations of the tool at each severity level are required to achieve inter-rater reliability [38]. Trial investigators are not trained to this level of reliability, and data collected exhibit large standard deviations and confidence intervals.

Studies have also shown substantial variability in trialto-trial instructions for both the ADAS-cog [35] and the NPI [36]. This variability causes uncertainty among trial site personnel as they encounter the same scale administered in different ways by varying sponsors. This increases score variability and undermines the ability to demonstrate a drug-placebo difference of effective agents.

Once trained, raters may not continue to rigorously adhere to scoring conventions and administration standards. In one international study with a ratings surveillance program in place, half of ADAS-cog raters and one-third of Mini-Mental Status Examination raters required some level of remediation after the conclusion of rater training [37]. Such programs are not in place for all trials and rater drift may affect outcomes.

These studies demonstrate deficiencies in training and test application in trials. These problems are not unique to international trials but are amplified in global trials where differences in language and trial site experience may be more marked and further undermine data reliability. Greater attention to rater training and rater monitoring or use of centralized raters may assist in managing measurement-related variability in AD clinical trials.

Good Clinical Practice guidelines have been incorporated into trial procedures to a variable extent across international sites. This leads to variations in the care with which informed consent is obtained, diligence in application of inclusion and exclusion criteria, rigor of collecting information on concomitant medications and use of over-the-counter treatments, attention to timeframes of data collection, thoroughness of adverse event reporting, and completeness of data collection and recording [37]. This variable familiarity with and application of good clinical practice may affect data quality.

Protection of human subjects and obtaining informed consent through provision of complete information to patients and caregivers and insuring that they understand the procedures, risks, and expectations of clinical trial participation is an essential and required aspect of $\mathrm{AD}$ clinical trial conduct. In countries with less extensive histories of research conduct and investigations involving human subjects, there may be less familiarity with these procedures. Institutional review boards may be inexperienced and understaffed $[37,39]$. This exposes the study to uninformed patient participation, lack of protection of human rights, unacknowledged conflicts of interest, and violations that may compromise study validity and acceptability.

Pharmacovigilance, both during trials and after drug approval in any country, provides critical data on drug safety and mechanisms must be in place to share this information globally in a timely way with appropriate translations and mechanisms for notifying local institutional review boards and patients.

\section{Extrapolation of clinical trial data among regions}

Guidelines regarding the proper sample size and statistical approaches to multi-regional trials are evolving. ICH guidelines [4] provide general instructions regarding extrapolation of data between regions and when bridging studies will be required. Tsou and colleagues [40] suggest that consistency of responses across ethnic groups in global trials is an important means of determining if responses can be extrapolated across regions. Ethnic groups or nations must comprise at least $20 \%$ of the trial population to make such analyses feasible [40]. Consistency in analyses can also be applied to determine if trials conducted in specific regions have outcomes consistent with reference trials conducted for regulatory approval in other countries [41].

Bridging studies are required by regulatory bodies when the validity of extrapolation of data from one region to another is uncertain. Bridging studies can vary from demonstration of safety in the new region, to doseresponse pharmacokinetic studies, to comprehensive clinical trials with requirements to demonstrate treatment efficacy $[42,43]$. Drugs less sensitive to ethnic factors (Table 4) are less likely to require bridging studies. Bridging studies can be conducted simultaneously with pivotal trials in global drug development programs to reduce delay in seeking marketing authorization in several countries at study conclusion [42].

\section{Regulatory factors that may vary among countries}

The ICH guideline E5 provides a framework for considering ethnic influences in global clinical trials [9]. The 
purpose of this guideline was to minimize unnecessary duplication of trials, save human and material resources, and expedite the development of medications to benefit patients [44]. This guidance is the major regulatory reference for sponsors and contract research organizations conducting international clinical trials.

For industry sponsors pursuing marketing authorization in international markets, strategies and requirements differ by country. Japan generally requires that 50 to $60 \%$ of patients in a trial be Japanese for the agent to be considered for regulatory approval there. China requires that 200 to 300 Chinese patients be included in a trial [23].

The US Food and Drug Administration (FDA) accepts foreign data in support of a marketing application for a human drug, biological product, or device as long as the data were collected in a study conforming to the ethical principles contained in the Declaration of Helsinki [45]. It is anticipated that the number of US patients in a pivotal trial comprising part of a New Drug Application (NDA) will permit analysis to determine if the agent was effective in the US population.

Data from international trial sites are increasingly included in marketing applications for drugs and biologics. In $2008,80 \%$ of applications included at least some data from foreign clinical trials [2]. The rise in the number of trials conducted abroad has not been matched with FDA regulatory scrutiny. An Office of the Inspector General review found that the FDA conducted inspections on only $0.7 \%$ of foreign clinical trial sites [46]. The results of these reviews suggested that international sites often do not meet expected clinical trial conduct criteria.

\section{Clinical operations and sample handling that may affect global trials}

Contractual relationships may vary from country to country and operational flexibility is required for global clinical trials. Administrative and financial arrangements also differ among countries.

Biomarker capacity may vary markedly among global sites. The US, Canada, and Western Europe tend to be early adopters of new technology compared to other countries, and advanced imaging capability will typically not be as widely available as clinical expertise in global sites. Use of phantoms for image standardization and machine calibration may not be familiar to all sites, and sponsors of international trials will need to provide additional technical expertise to ensure quality [47]. Use of centralized reading of brain imaging is advantageous to minimize site-to-site variability in image interpretation.

Sample collection and handling may also pose challenges in global trials. China, for example, has restrictions on DNA and plasma export, requiring identification and standardization of a reference laboratory in China in order to pursue trials in that country.
Maintaining supply line integrity can be difficult in international circumstances. Importation laws can slow movement of drugs across international borders and implementing Good Manufacturing Practices for local production can take valuable time and delay trial initiation.

\section{Comment}

Globalization of $\mathrm{AD}$ clinical trials is a consistent trend over the past decade and will continue into the foreseeable future [7]. Including international sites in AD trials allows timely patient recruitment, diversification of trial participants (including patients more likely to be treatment naïve), conduct of trials with less expense and facilitation of marketing applications in participating countries. Global trials have advantages for patients, investigators, and sponsors. Patients get access to experimental agents and may experience a higher level of care than is otherwise available; medications may become available in international markets more rapidly. Investigators are able to participate in international collaborations, contribute to the advancement of therapies and scientific progress, increase skill levels of themselves and their teams, may augment income and may experience increased prestige and credibility. Sponsors benefit from cost savings, speed of recruitment, and market access. These advantages must be considered in the context of the many challenges to conducting clinical trials on a global scale, including uncertainties regarding differences in disease biology, the effect of ethnicity on drug pharmacokinetics and pharmacodynamics, and challenges to adequate human protections.

Attention to the factors discussed (Table 3 ) is necessary to ensure the integrity of data included in multiregional trials. Many issues that may affect global trials have not been studied in sufficient detail to predict the magnitude of the effect on trial outcomes. Some issues such as trial conduct can be addressed by rigorous site training and scrupulous monitoring and oversight during the course of the trial. Central ratings and application of new assessment technologies, such as computerized data collection, may improve some aspects of data quality. Biological factors that may be related to ethnicity and may affect pharmacokinetics, pharmacodynamics, and disease biology are more difficult to address. More rigorous scientific investigation of these factors and their effects in trials is needed. Complex modeling may be needed to determine the effects of education and head size on disease progression, for example; such models may determine adjustments in sample size or trial duration needed for efficacy trials.

Scrutiny of the factors affecting international trials will help avoid failed trials and facilitate the development of urgently needed new treatments for $\mathrm{AD}$. 


\section{Conclusion}

Intrinsic biological and extrinsic sociocultural factors may affect trial outcomes in AD drug development programs. Host, disease and trial conduct practices may all vary internationally. Cross-national comparison studies are few and the effect of regional variations has been inadequately explored. Attention to these factors is important to successful global trials.

\section{Abbreviations}

AD, Alzheimer's disease; ADAS-cog, Alzheimer's Disease Assessment Scale - Cognitive portion; FDA, Food and Drug Administration; ICH, International Conference for Harmonization; NPI, Neuropsychiatric Inventory.

\section{Competing interests}

JC has provided consultation to Abbott, Acadia, Acerra, ADAMAS, Anavex, Astellas, Avanir, Baxter, Bristol-Myers Squibb, Eisai, Elan, EnVivo, Forest, Genentech, GlaxoSmithKline, Janssen, Lilly, Lundbeck, Medivation, Medtronics, Merck, Merz, Neurokos, Novartis, Pfizer, Prana, QR, Sonexa, Takeda, and Toyama pharmaceutical companies. JC has also provided consultation to Bayer, Avid, GE, MedAvante, Neurotrax, and UBC. JC owns stock in ADAMAS, Prana, Sonexa, MedAvante, Neurotrax, Neurokos, and QR pharma. JC has acted as a speaker for Eisai, Forest, Janssen, Novartis, Pfizer and Lundbeck. JC owns the copyright of the Neuropsychiatric Inventory and has provided expert witness consultation regarding olanzapine and ropinerol. KZ has consulted for Healthcare Communications Group, Baxter and Pfizer Pharmaceuticals. RR has no disclosures.

\section{Acknowledgements}

The authors acknowledge Christine Moore of Cleveland Clinic for technica assistance in manuscript preparation.

\section{Author details}

'Cleveland Clinic Lou Ruvo Center for Brain Health, Las Vegas, Nevada 89106, USA. ${ }^{2}$ Tuoro School of Osteopathic Medicine, Las Vegas, Nevada 89074, USA.

\section{Published: 17 August 2011}

\section{References}

1. Wimo A, Prince M: World Alzheimer Report 2010: The Global Economic Impact of Dementia. London: Alzheimer's Disease International; 2010.

2. Phelps K, Kumar V: A new global view. The use of foreign data in INDs requires rethinking clinical trials. Drug Discov Dev 2011, January:30-32

3. Maiti RMR: Clinical trials in India. Pharm Res 2007, 56:1-10.

4. Chui KFK, Lam LCW: Relevance of outcome measures in different cultural groups - does one size fit all? Internat Psychoget 2007, 19:457-466.

5. World Health Organization: The World Medicines Situation. Geneva: World Health Organization; 2004.

6. Glickman SW, McHutchinson JG, Peterson ED, Cairns CB, Harington RA, Calif RM, Schulman KA: Ethical and scientific implications of the globalization of clinical research. N Eng J Med 2009, 360:816-823.

7. Karlberg JPE: The Establishment of Emerging Trial Regions. Clinical Trial Magnifier 2011, 4:7-23.

8. Cummings $\mathrm{J}$ : What can be inferred from the interruption of the semagacestat trial for treatment of Alzheimer's disease? Biol Psychiatry 2010, 68:876-878.

9. Food and Drug Administration: International Conference on Harmonisation; Guidance on Ethnic Factors in the Acceptability of Foreign Clinical Data; Availability. Federal Register 1998, 63:31790-31796.

10. Human Development Report: The Real Wealth of Nations: Pathways to Human Development. United Nations Development Program; 2010.

11. Wu CC, Yeh WT, Crow RS, Bai CH, Pan WH: Comparison of electrocardiographic findings and associated risk factors between Taiwan Chinese and US White adults. Int J Cardiol 2008, 128:224-231.

12. Education Level Statistics [http://www.nationmaster.com/index.php]

13. Wang H, Yu X, Li S, Chen Y, Li H, He J: The Cognitive Subscale of Alzheimer's Assessment Scale, Chinese version in staging of Alzheimer disease. Alz Dis Assoc Disord 2004, 18:231-235.

14. Petersen RC, Aisen PS, Beckett LA, Donohue MC, Gamst AC, Harvery DJ, Jack
CR Jr, Jugust WJ, Shaw LM, Toga AW, Trojanowski JQ, Weiner MW: Alzheimer's Disease Neuroimaging Initiative (ADNI): clinical characterization. Neurology 2010, 74:201-209.

15. Stern Y, Albert S, Tang MX, Tsai WY: Rate of memory decline in AD is related to education and occupation. Neurology 1999; 53:1942-1947.

16. Bennett DA, Wilson RS, Schneider JA, Evans DA, Mendes de Leon CF, Arnold SE, Barnes LL, Bienias JL: Education modifies the relation of AD pathology to level of cognitive function in older persons. Neurology 2003, 60:1909-1915.

17. Roe CM, Mintun MA, D'Angelo G, Xiong C, Grant EA, Morris JC: Alzheimer disease and cognitive reserve. Arch Neurol 2008, 65:1467-1471.

18. Roe CM, Xiong C, Miller JP, Cairns NJ, Morris JC: Interaction of neuritic plaques and education predicts dementia. Alzheimer Dis Assoc Disord 2008, 22:188-193.

19. Kemppainen NM, Aalto S, Karrasch M, Nagren K, Savisto N, Oikonen V, Parkkola R, Rinne J: Cognitive reserve hypothesis: Pittsburgh compound b and fluorodeoxyglucose positron emission tomography in relation to education in mild Alzheimer's disease. Ann Neurol 2008, 63:112-118.

20. Liang KY, Mintun MA, Fagan AM, Goate AM, Bigg JM, Holtzman DM, Morris JC, Head D: Exercise and Alzheimer's disease biomarkers in cognitively normal older adults. Ann Neurol 2010, 68:311-318.

21. Case A, Paxson C: Stature and status: height, ability and labor market outcomes. J Polit Econ 2008, 116:499-532.

22. Ichimaru K, Toyoshima S, Uyama Y: Effective global drug development strategy for obtaining regulatory approval in Japan in the context of ethnicity-related drug response factors. Clin Pharm Ther 2010, 87:362-366.

23. Kudrin $\mathrm{A}$ : Challenges in the clinical development requirements for the marketing authorization of new medicines in Sutheast Asia. J Clin Pharmacol 2009, 49:268-280.

24. Bjornsson TD, Wagner JA, Donahue SR, Harper D, Karim A, Khouri M, Murphy WR, Roman K, Schneck D, Sonnichsen DS, Stalker DJ, Wise SD, Dombey S, Loew C: A review and assessment of potential sources of ethnic differences in drug responsiveness. J Clin Pharmacol 2003, 43:943-967.

25. Faison WE, Schultz SK, Aerssens J, Alvidrez J, Anand R, Farrer LA, Jarvik L, Manly J, McCrae T, Murphy GM Jr, Olin JT, Regier D. Sano M, Mintzer JE: Potential ethnic modifiers in the assessment and treatment of Alzheimer's disease: challenges for the future. Internat Psychoget 2007, 19:539-558.

26. Singh PP, Singh M, Mastana SS: APOE distribution in world populations with new data from India and the UK. Ann Human Biol 2006, 33:279-308.

27. Okonkwo OC, Alosco ML, Jerskey BA, Sweet LH, Ott BR, Tremont G, the Alzheimer's Disease Neuroimaging Initiative: Cerebral atrophy, apolipoprotein $\mathrm{E} \varepsilon 4$ and rate of decline in everyday function among patients with amnestic mild cognitive impairment. Alzheimer's Dement 2010, 6:404-411.

28. Blom ES, Giedratis V, Zetterberg H, Fukumoto H, Blennow K, Hyman B, Irizarry MC, Wahlund LO, Lannfelt L, Ingelsson M: Rapid progression from mild cognitive impairment to Alzheimer's disease in subjects with elevated levels of tau in cerebrospinal fluid and the APOE $\varepsilon 4 / \varepsilon 4$ genotype. Dement Geriatr Cogn Disord 2009, 27:458-464.

29. Graves AB, Mortimer JA, Larson EB, Wenzlow A, Bowen JD, McCormick WC: Head circumference as a measure of cognitive reserve. Association with severity of impairment in Alzheimer's disease. Br J Psychiatry 1996, 169:86-92.

30. Whitwell JL: The protective role of brain size in Alzheimer's disease. Expert Rev Neurother 2010, 10:799-801.

31. Binetti G, Mega MS, Magni E, Padovani A, Rozzini L, Lussignoli G, Bianchetti A Trabucchi M, Cummings J: Behavioral disorders in Alzheimer's disease: a transcultural perspective. Arch Neurol 1998, 55:539-544

32. Sekita A, Ninomiya T, Tanizaki Y, Doi Y, Hata J, Yonemoto K, Arima H, Sasaki K, lida M, Iwaki T, Kanba S, Kiyohara Y: Trends in prevalence of Alzheimer's disease and vascular dementia in a Japanese community: the Hisayama Study. Acta Psychiatr Scand 2010, 122:319-325.

33. Chow TW, Liu CK, Fuh JL, Leung VP, Tai CT, Chen LW, Wang SJ, Chiu HF, Lam LC, Chen QL, Cummings JL: Neuropsychiatric symptoms of Alzheimer's disease differ in Chinese and American patients. Int J Geriatr Psychiatry 2002, 17:22-28.

34. Diaz SPR, Gregorio PG, Casdado JMR, Reynich E, Ousset PJ, Vellas B, Salmon E, and EADC centers and investigators: The need for a consensus in the use of assessment tools for Alzheimer's disease: the feasibility study (assessment) tools for dementia in Alzheimer Centres across Europe), a European Alzheimer's Disease Consortium (EADC) survey. Int J Geriatr 
Psychiatry 2005, 20:744-748.

35. Connor DJ, Sabbagh MN: Administration and scoring variance on the ADAS-cog. J Alzheimers Dis 2008, 15:461-464.

36. Connor DJ, Sabbagh MN, Cummings JL: Comment on administration and scoring of the Neuropsychiatric Inventory in clinical trials. Alzheimer's Dem 2008, 4:390-394.

37. Doody RS, Cole PE, Miller DS, Siemers E, Black R, Feldman H, Schindler R, Graham S, Heath T, Khachaturian AS, Evans R, Carrillo MC: Global issues in drug development for Alzheimer's disease. Alzheimers Dement 2011 7:197-207.

38. Tractenberg RE, Yumoto F, Jin S, Morris JC: Sample size requirements for training to a k agreement criterion on clinical dementia ratings. Alz Dis Assoc Disord 2010, 24:264-268.

39. US Department of Health and Human Services - Office of Inspector General: The Globalization of Clinical Trials. A Growing Challenge in Protecting Human Subjects. OEl-01-0-00190. September 2001:1-51.

40. Tsou H-H, Chow S-C, Lan G, Liu J-p, Wang M, Chern H-D, Ho L-T, Hsiung CA, Hsaio C-F: Proposals of statistical consideration to evaluation for a specific region in multi-regional trials - Asian perspective. Pharm Stat 2010, 9:201-206.

41. Shih WJ: Clinical trials for drug registration in Asian-Pacific countries: proposal for a new paradigm from a statistical perspective. Controlled Clin Trials 2001, 22:357-366.
42. US Department of Health and Human Services - Food and Drug Administration: Guidance for Industry: E5 - Ethnic Factors in the Acceptability of Foreign Clinical Data, Questions, and Answers. September 2006:1-8.

43. European Medicines Agency: ICH Topic e (R1): Note for Guidance on Ethnic Factors in the Acceptability of Foreign Clinical Data - Step 5. September 1998:1-15.

44. Goto M, Hamasaki T: Practical issues and observations on the use of foreign clinical data in drug development. J Biopharm Stat 2002, 12:369-384.

45. US Department of Health and Human Services - Food and Drug Administration: Guidance for Industry: Acceptance of Foreign Clinical Studies. March 2001:1-2.

46. US Department of Health and Human Sevices - Office Inspector General: Challenges to FDA's Ability to Monitor and Inspect Foreign Clinical Trials. OEl-01-08-00501. June 2010:1-43.

47. Schindler RJ: Study design considerations: conducting global clinical trials in early Alzheimer's disease. J Nutr Health Aging 2010, 14:312-314.

\section{doi:10.1186/alzrt86}

Cite this article as: Cummings J, et al.: Globalization of Alzheimer's disease clinical trials. Alzheimer's Research \& Therapy 2011, 3:24. 